\title{
MODELOS MATEMÁTICOS PREDADOR-PRESA E APLICAÇÕES AO MANEJO INTEGRADO DE PRAGAS
}

\author{
Ana Paula M. B. Battel ${ }^{I^{*}}$, Rafael A. Moral ${ }^{1,2} \&$ Wesley A. C. Godoy ${ }^{1}$ \\ ${ }^{1}$ Universidade de São Paulo (USP), Escola Superior de Agricultura Luiz de Queiroz (ESALQ), Departamento de Entomologia e Acarologia. Avenida \\ Pádua Dias, no:11, Piracicaba, São Paulo, Brasil. CEP: 13418-900. \\ ${ }^{2}$ Universidade de São Paulo (USP), Escola Superior de Agricultura Luiz de Queiroz (ESALQ), Departamento de Ciências Exatas. Avenida Pádua Dias, \\ $\mathrm{n}^{\circ}: 11$, Piracicaba, São Paulo, Brasil. CEP 13418-900. \\ E-mails: ap_mb@hotmail.com,rafa_moral2004@yahoo.com.br,wacgodoy@usp.br
}

\section{RESUMO}

A modelagem matemática de sistemas predador-presa e hospedeiro-parasitóide é uma ferramenta importante para descrever as interações entre pragas e inimigos naturais em programas de controle biológico. Os modelos predador-presa desenvolvidos por Lotka e Volterra vêm sendo aprimorados, com a inserção de parâmetros relevantes a cada tipo de estudo. Dentre as inserções realizadas em modelos, abordagens direcionadas para o manejo integrado de pragas têm sido implementadas para avaliar o efeito de inseticidas sobre a dinâmica predador-presa. É possível inserir os efeitos da aplicação de inseticidas e avaliar o comportamento dinâmico resultante dessa aplicação em presas e predadores ou hospedeiros e parasitóides. Também se pode atribuir estocasticidade aos parâmetros do modelo, a fim de se obter simulações mais próximas à realidade, levando em conta os efeitos das variações não-controláveis. O desenvolvimento de novos modelos é encorajado nesta revisão, a fim de que os efeitos dos principais fatores que influenciam as dinâmicas em agroecossistemas sejam modelados mais precisamente.

Palavras-chave: Modelagem matemática de manejo de pragas; modelos predador-presa.

\section{ABSTRACT}

PREDATOR-PREY MATHEMATICAL MODELS AND APPLICATIONS ON INTEGRATED PEST MANAGEMENT. Mathematical modeling of predator-prey and host-parasitoid systems is an important tool for describing the interactions among pests and natural enemies in biological control programs. Predator-prey models developed by Lotka and Volterra have been improved with the insertion of relevant parameters for each type of study. Among the insertions made in models, approaches directed towards the integrated pest management have been implemented to evaluate the effect of insecticides in the predator-prey dynamics. It is possible to infer the effects of insecticide application and evaluate the dynamic behavior resultant of this application in prey, predator or host and parasitoid. One could also add stochasticity to the models' parameters, in order to obtain dynamic behavior simulations which are closer to reality, with the non-controllable variation effects taken into account. The development of new models is encouraged in this review, so as to the effects of the main factors which influence the dynamics in agroecosystems are more precisely modeled.

Keywords: Pest management mathematical model; predator-prey models.

\section{RESUMEN}

MODELOS MATEMATICOS PREDADOR-PRESA Y APLICACIONES EM LOS PLANES DE MANEJO INTEGRADO DE PLAGAS. El modelado matemático de sistemas predador-presa y hospedero-parasitoide es una herramienta importante utilizada para describir las interacciones entre plagas y enemigos naturales en programas de control biológico. Los modelos predador-presa desarrollados por Lotka y Volterra han sido mejorados con la inserción de parámetros relevantes para cada tipo de estudio. 
Dentro de las inserciones realizadas en los modelos, los abordajes direccionados para el manejo integrado de plagas también han sido implementados para evaluar el efecto de insecticidas sobre la dinámica predador-presa. Es posible inferir los efectos de la aplicación de insecticidas y evaluar el comportamiento dinámico resultante de esa aplicación en presas y predadores u hospederos y parasitoides. También se puede atribuir estocasticidad a los parámetros del modelo a fin de obtener simulaciones más próximas a la realidad, teniendo en cuenta los efectos de las variaciones no controlables. El desarrollo de nuevos modelos es alentado en esta revisión, a fin de que los efectos de los principales factores que influencian las dinámicas en los agroecosistemas, sean modelados con mayor precisión.

Palabras-clave: Modelo matemático de manejo de plagas; modelos predador-presa.

\section{INTRODUÇÃO}

Modelos matemáticos aplicados a sistemas ecológicos podem ser vistos como caricaturas idealizadas a partir da abstração de biossistemas, desenhadas para descrever padrões geralmente de difícil visualização apenas pela inspeção visual de dados (Lima et al. 2009). Os modelos que buscam a descrição de interações entre espécies têm como meta capturar a essência dos processos biológicos envolvidos no sistema, utilizando para tanto formalismos matemáticos que incluem funções adequadas para explicitar a dimensão correta do comportamento das populações em sistemas dinâmicos (Kot 2001). As funções utilizadas geralmente incluem desde simples relações lineares até complexos formatos de natureza nãolinear (Prout \& McChesney 1985, Cushing et al. 2003). Os modelos que incorporam interações interespecíficas incluem proposições interessantes para descrever a dinâmica de sistemas envolvendo diferentes espécies, tais como, competidores, predadores, presas, hospedeiros, parasitóides e parasitas (Kot 2001). Tomando como base o formalismo teórico clássico, é natural que, no contexto de agroecossistemas, o foco da atenção esteja sobre os modelos do tipo predador-presa ou hospedeiro-parasitóide, sobretudo quando se pensa em enfatizar estratégias de controle biológico de pragas (Murdoch et al. 1985).

Nesta revisão, alguns modelos matemáticos são apresentados de forma a historiar a trajetória do pensamento sobre modelagem ecológica aplicada ao manejo de pragas. O estudo relata desde as bases ecológicas que inicialmente deram sustentação aos modelos do tipo predador-presa, até a combinação com funções matemáticas capazes de inserir diferentes estratégias de controle de pragas nas formulações. A revisão é finalizada com detalhada análise do formalismo proposto por Hassell (1984), com vistas à comparação de quatro cenários de manejo de pragas, os quais pressupõem a ação de inseticidas sobre a dinâmica predadorpresa ou hospedeiro-parasitóide em diferentes instantes de aplicação e atingindo parcialmente ou integralmente os componentes do modelo interativo. A fundamentação teórica inerente dos modelos de Lotka-Volterra foi apresentada como estrutura básica para a proposição de formalismos teóricos voltados para o controle de pragas. Contudo, a teoria ecológica foi revisitada visando o aprimoramento da abordagem teórica aplicada à modelagem do manejo integrado de pragas.

\section{MODELOS PREDADOR-PRESA}

Lotka (1925) e Volterra (1926) foram os precursores da idéia de investigar as relações entre predadores e presas assumindo, inicialmente, premissas extremamente simples, porém capazes de produzir visualização do que é primordial para o sistema. A proposição pressupunha que, exceto pela presença de predadores, presas poderiam sobreviver satisfatoriamente em ambientes desprovidos de limitações de recursos e, por sua vez, predadores seriam limitados somente pela disponibilidade de presas. O modelo preliminar foi assim proposto para descrever um sistema que se autosustentaria por tempo indeterminado somente com estas simplificações, gerando ciclos periódicos (Figura 1). Contudo, para viabilizar este processo outras pressuposições foram feitas, como por exemplo, a reprodução contínua de predadores e presas produzindo indivíduos idênticos e a taxa 
de predação sendo proporcional à taxa de encontro entre os componentes do sistema, com função aleatória da densidade populacional (Costa \& Godoy 2010). As equações (1) e (2) descrevem o processo de predação proposto por Lotka \& Volterra.

$$
\begin{gathered}
\frac{d p}{d t}=r p-f p P \\
\frac{d P}{d t}=-m P+n f p P
\end{gathered}
$$

Nas equações acima, a trajetória da população de presas $(p)$ e de predadores $(P)$ seguem em tempo contínuo, de acordo com as equações diferenciais. A população de presas (Equação 1) depende da sua taxa intrínseca de crescimento $(r)$ e do seu número inicial de indivíduos $(p)$. A taxa de encontro entre $p$ e $P$ é governada por $f$, comumente denominada de resposta funcional do predador. Assume-se, para este caso simplificado, que essa constante varia linearmente de acordo com a abundância de presas. A equação para predadores (Equação 2) descreve o crescimento populacional pressupondo que $m$ é a mortalidade da população de predadores na ausência da população de presas. A resposta numérica, definida como a eficiência de conversão de presas em predadores, é dada por $n$, que mede a produção per capita de prole de predadores também como uma função da abundância de presas.

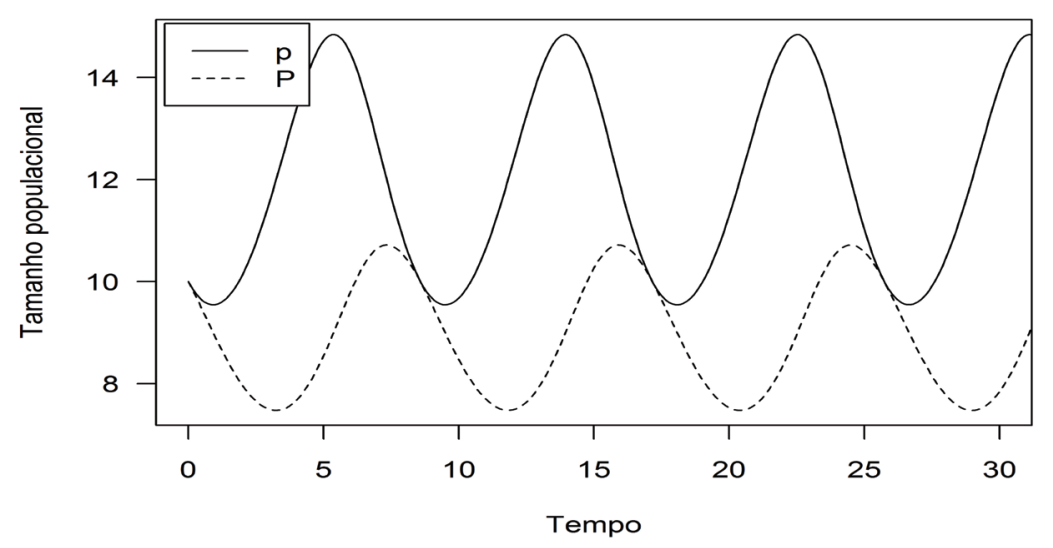

Figura 1. Recorrência temporal para o modelo predador-presa em tempo contínuo. Valores dos parâmetros usados na simulação: $r=0.9, f=0.1, m=0.6$, $n=0.5, p=10, P=10$.

Figure 1. Recurrence plot for the time series generated by the continuous time predator-prey model. Parameter values used in the simulation: $r=0.9$, $f=0.1, m=0.6, n=0.5, p=10, P=10$.

A inclusão da dependência da densidade no modelo descrito pelas equações (1) e (2) pode ser obtida pela modificação da equação (1) resultando em:

$$
\frac{d p}{d t}=r p\left(1-\frac{p}{K}\right)-f p P
$$

Com a dependência da densidade inserida na equação da presa, o sistema é estabilizado, deixando de exibir as oscilações periódicas (Figura 2). Voltando às equações (1) e (2), resta ainda investigar os aspectos relacionados à resposta funcional. A resposta funcional é uma relação que estima a saturação do consumo de presas pelo predador (Holling 1965), podendo convencionalmente assumir três formatos (Figura 3), linear (tipo I), assintótico (tipo II) e sigmóide (tipo III). Os formatos mais comuns encontrados em agrossistemas, enfatizando a dinâmica populacional de pragas, são o assintótico e o sigmóide (De Clercq et al. 2000). Assumindo que $p_{a}$ determina o número de presas atacadas, postulase que este termo depende essencialmente da taxa de ataque de um único predador sobre suas presas $(a)$, do tempo de procura por presas $\left(T_{p}\right)$ e também da densidade de presas $(p)$. A equação que representa esta dependência pode então ser escrita como:

$$
p_{a}=a T_{p} p
$$




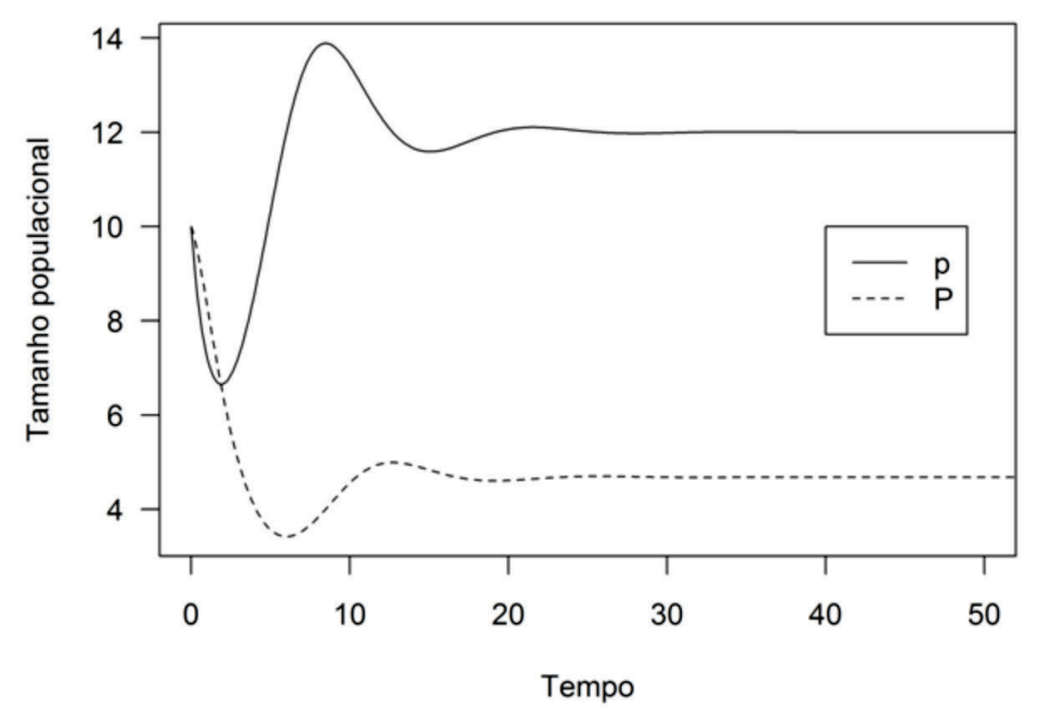

Figura 2. Recorrência temporal para o modelo predador-presa em tempo contínuo com dependência da densidade incluída. Valores dos parâmetros usados na simulação: $r=0.9, f=0.1, m=0.6, n=0.5, K=25, p=10, P=10$.

Figure 2. Recurrence plot for the time series generated by the continuous time predator-prey model with included density dependence. Parameter values used in the simulation: $r=0.9, f=0.1, m=0.6, n=0.5, K=25, p=10, P=10$.

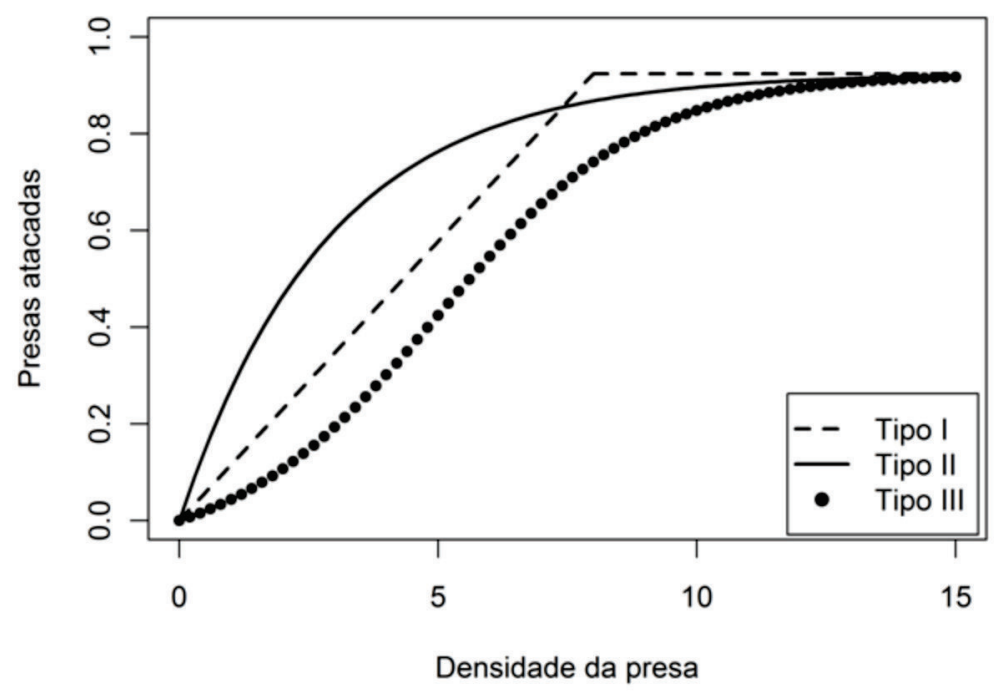

Figura 3. Resposta funcional do tipo linear (tipo I), assintótico (tipo II) e sigmóide (tipo III).

Figure 3. Linear (type I), asymptotic (type II) and sigmoid (type III) functional responses.

Assumindo que cada presa consumida demanda algum tempo de manipulação $\left(T_{m}\right)$, este período de tempo deve ser subtraído do tempo total $\left(T_{t}\right)$,

$$
T_{p}=T_{t}-T_{m} p_{a}
$$

Substituindo (5) em (4), tem-se:

$$
p_{a}=a p\left(T_{t}-T_{m} p_{a}\right)
$$

que, rearranjada, resulta em:

$$
p_{a}=\frac{a p T_{t}}{1+a p T_{m}}
$$

Com base nisso, o número de presas atacadas por unidade de tempo é:

$$
\frac{p_{a}}{T_{t}}=\frac{a p}{1+a p T_{m}}
$$


Incluindo a resposta funcional nas equações de dinâmica para presas e predador, obtém-se:

$$
\begin{gathered}
\frac{d p}{d t}=r p-\frac{a p}{1+a p T_{m}} P \\
\frac{d P}{d t}=-m P+n \frac{a p}{1+a p T_{m}} P
\end{gathered}
$$

\section{MODELOS HOSPEDEIRO-PARASITÓIDE}

As formulações teóricas pioneiras para estudar a dinâmica de pragas em agrossistemas foram propostas por Nicholson \& Bailey (1935), que partiram de pontos básicos para investigar sistemas dinâmicos mais complexos. A teoria foi fundamentada em duas simplificações: (1) o encontro $(E)$ entre parasitóides ou predadores $(P)$ com hospedeiros ou presas $(p)$ deveria ser proporcional à densidade de hospedeiros ou presas e (2) os encontros seriam aleatoriamente distribuídos entre as presas. Esta função pode ser descrita pelo termo zero da distribuição de Poisson, escrita como

$$
p_{0}=\exp \left(\frac{-E}{p}\right)
$$

e o número de presas (ou hospedeiros) atacadas (ou parasitadas) assume a forma:

$$
p_{a}=p\left[1-\exp \left(\frac{-E c}{p}\right)\right]
$$

Então, o encontro entre predadores $P$ e presas $p$ pode ser expresso por

$$
E=a p P \quad(13),
$$

onde $a$ é uma constante de proporcionalidade denominada área de captura pelo predador. Tratase de uma estimativa da eficiência de encontro. Assim, $E_{c} / p=a P$ e o número de presas consumidas pode ser escrito como

$$
p_{a}=p[1-\exp (-a P)]
$$

A equação (14) denota que a predação ou parasitismo aumentará até determinado ponto de saturação $a P$, visto que a taxa de encontro entre predadores e presas diminui à medida que a eficiência de encontro também aumenta. Isto vem da conclusão que o número de presas disponíveis diminui em decorrência da boa eficiência de encontro de presas pelo predador. $\mathrm{O}$ modelo geral para descrever a dinâmica predador-presa incluindo a resposta funcional é demonstrado pelas equações (9) e (10) e aparece no formato de equações diferenciais para representar um sistema contínuo. Contudo, muitos sistemas como, por exemplo, aqueles em que os indivíduos se reproduzem sem sobreposição de gerações, são melhores descritos por equações em tempo discreto. Nesse caso, as equações recursivas assumem o formato:

$$
\begin{gathered}
p_{t+1}=\psi p f(p, P) \\
P_{t+1}=p[1-f(p, P)]
\end{gathered}
$$

onde $f(p, P)=\exp (-a P)$ determina o número de presas não atacadas ou de hospedeiros não parasitados. O parâmetro $\psi$ representa a taxa intrínseca de crescimento geométrico para presas. Com este formato, a dinâmica predador-presa seria descrita por um padrão linear de resposta funcional (tipo I). Para descrever a dinâmica do sistema com resposta funcional assintótica (tipo II), basta assumir

$$
f(p, P)=\exp \left(\frac{-a T P}{1+a T_{m} p}\right)
$$

Na equação (17) $T_{m}$ determina o tempo de manipulação que um predador requer sobre a presa, $a^{\prime}$ é a taxa de ataque e $T$ representa o tempo total. Em altas densidades de presa, o tempo utilizado pelo predador para consumir a presa deve ser otimizado, o que pode levar à saturação da resposta funcional, traduzida pela resposta assintótica, conhecida como do tipo II. Quando $a$ e $T_{m}$ são constantes e a busca por presas é aleatória, a função que determina a sobrevivência das presas é

$$
f(p, P)=\exp \left(\frac{-a T P}{1+a T_{m} p}\right)
$$

A resposta sigmóide (tipo III) aparece quando um ou mais parâmetros associados à captura de presas ( $a, T$, ou ambos) são funções crescentes da densidade de presas. Já a função de sobrevivência das presas para a resposta do tipo III pode ser escrita como:

$$
f(p, P)=\exp \left(\frac{-b_{1} T p P}{1+b_{2} p+b_{1} T_{m} p^{2}}\right)
$$


Nesta função, $b_{1}$ e $b_{2}$ são constantes, sendo que $b_{2}$ denota a resposta numérica do predador, ou seja, o número de presas convertidas em prole. Em parasitóides, é comum atribuir 1 para $b_{2}$, visto que as fêmeas do inseto geralmente obtém apenas um indivíduo (em termos de prole) por hospedeiro. A dinâmica populacional de um sistema predadorpresa incluindo a resposta funcional assintótica (tipo II) poderia ser descrita pela recorrência que aparece na Figura 4.

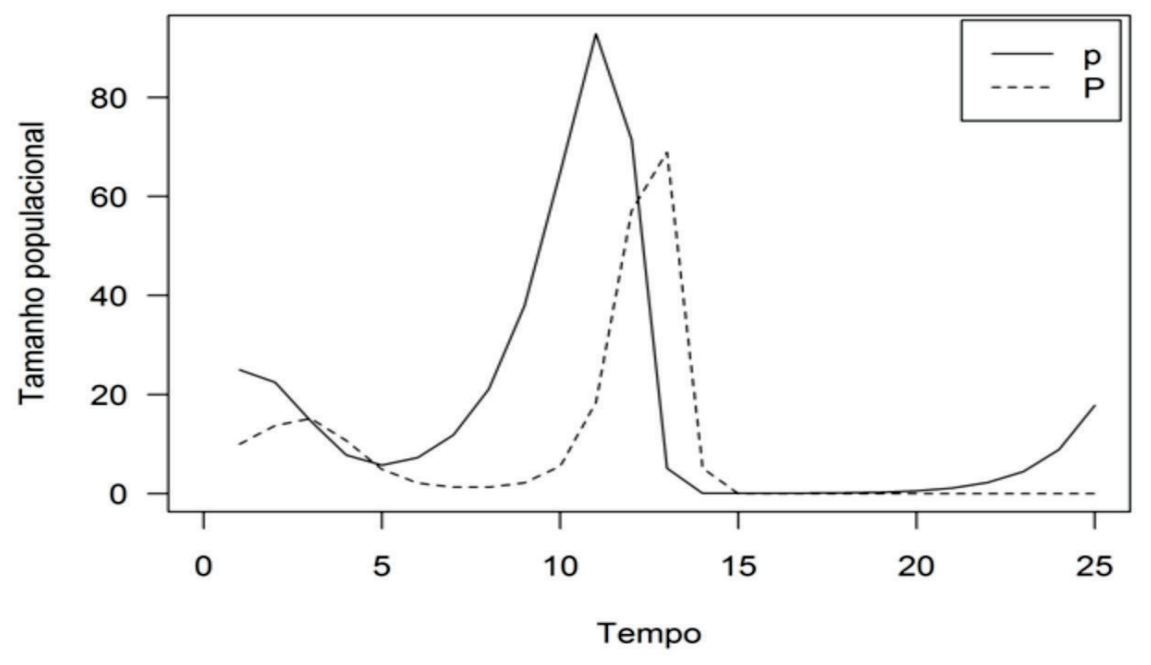

Figura 4. Recorrência no tempo para a dinâmica predador-presa com resposta funcional do tipo II. Valores dos parâmetros usados na simulação: $\psi=2$, $T_{m}=0.1, T=1, a=0.1, p=25, P=10$.

Figure 4. Recurrence plot for the time series generated by the predator-prey dynamics with type II functional response. Parameter values used in the simulation: $\psi=2, T_{m}=0.1, T=1, a=0.1, p=25, P=10$.

\section{INCORPORANDO O EFEITO DE INSETICIDAS}

\section{MODELOS DE HASSEL (1984)}

O que foi demonstrado até a equação (19) sintetiza de certa forma os fundamentos ecológicos para a aplicação de modelos matemáticos do tipo predador-presa em agroecossistemas, sobretudo quando se pensa em controle biológico de pragas. Contudo, raramente em agroecossistemas aplicam-se somente estratégias estritamente de controle biológico. Outros métodos de controle de pragas estão geralmente associados às práticas de controle, trazendo também a dimensão de manejo integrado de pragas MIP (Pedigo \& Zeiss 1996) para o arcabouço teórico aqui mencionado. O manejo integrado de pragas consiste de uma combinação de métodos de controle de pragas, usualmente desenvolvido para programas de longa duração. O programa de controle inclui estratégias capazes de combinar controle biológico, cultural, e aplicações de produtos químicos para reduzir populações de pragas a níveis toleráveis para a agricultura (Pedigo \& Zeiss 1996, van Lenteren 2000). Geralmente, os níveis são determinados por monitoramento periódico com fundamento no nível de dano econômico (Pedigo \& Zeiss 1996, Lima et al 2009).

Um estudo pioneiro na combinação entre modelo dinâmico para hospedeiro-parasitóide e outro método de controle de pragas, no caso, aplicação de inseticida, foi proposto por Hassell (1984). Em sua proposição, o autor analisa, do ponto de vista teórico, o equilíbrio e a estabilidade de um modelo que combina a dinâmica hospedeiro-parasitóide com os efeitos da aplicação de inseticida no sistema (Hassell 1984). O sistema foi investigado considerando-se quatro possibilidades: (1) inseticidas agindo antes do 
parasitismo e eliminando somente hospedeiros, (2) inseticidas agindo após o parasitismo e eliminando somente hospedeiros, (3) inseticidas agindo após o parasitismo e eliminando também os hospedeiros parasitados na mesma taxa que a possibilidade anterior e, finalmente, (4) inseticidas agindo antes do parasitismo e eliminando também parasitóides adultos.

O estudo proposto por Hassell (1984) manteve a mesma estrutura teórica empregada na função da resposta funcional tipo II (eq. 18). Contudo, a dimensão de aleatoriedade dada pela distribuição de Poisson apresentada na equação (11) foi substituída pela dimensão de agregação dada pela distribuição Binomial negativa, com média igual a $a P /\left(1+a T_{m} p\right)$. A função de sobrevivência de presas modificada pode ser escrita como:

$$
f(p, P)=\left[1+\frac{a P}{k\left(1+a T_{m} p\right)}\right]^{-k}
$$

O parâmetro $k$ (eq. 20) descreve o grau de agregação de predadores/parasitóides, sendo que quando $k$ tende à zero obtém-se o maior nível de agregação populacional (Southwood \& Henderson 2006). O processo de agregação espacial em populações biológicas tem sido considerado comum em diferentes espécies, com destaque para parasitas e insetos (Hassell 1980, Evans \& Toler 2007).

A inserção do termo que caracteriza a aplicação de inseticida antes da liberação de parasitóides que resulta na eliminação somente de hospedeiros (possibilidade 1) produz as recursões:

$$
\begin{gathered}
p_{t+1}=r p_{t} i f\left(p_{t}, i, P_{t}\right) \\
P_{t+1}=p_{t} i\left[1-f\left(p_{t}, i, P_{t}\right)\right]
\end{gathered}
$$

onde $i$ determina a probabilidade de um hospedeiro (ou uma presa) sobreviver à aplicação de inseticida.
Para tanto, a função de sobrevivência do hospedeiro pode ser escrita como:

$$
f\left(p_{t}, i, P_{t}\right)=\left[1+\frac{a P_{t}}{k\left(1+a \theta p_{t} i\right)}\right]^{-k}
$$

A inclusão do efeito do inseticida no sistema com ação após o parasitismo e eliminando somente hospedeiros (possibilidade 2) resulta em:

$$
\begin{array}{r}
p_{t+1}=r p_{t} f\left(p_{t}, P_{t}\right) i \\
P_{t+1}=p_{t}\left[1-f\left(p_{t}, P_{t}\right)\right]
\end{array}
$$

$\operatorname{com} f\left(p_{t}, P_{t}\right)$ definido na equação (20). Se a aplicação de inseticida for feita após o parasitismo, porém eliminando também os hospedeiros parasitados na mesma proporção que o caso anterior (possibilidade 3), as recursões serão escritas como:

$$
\begin{array}{r}
p_{t+1}=r p_{t} f\left(p_{t}, P_{t}\right) i \\
P_{t+1}=p_{t} i\left[1-f\left(p_{t}, P_{t}\right)\right]
\end{array}
$$

com $f\left(p_{p} P_{t}\right)$ também definido pela equação (20). Assumindo que o inseticida atue antes do parasitismo, porém eliminando também os parasitóides adultos (possibilidade 4) obtém-se:

$$
\begin{array}{r}
p_{t+1}=r p_{t} i f\left(p_{t}, i, P_{t}, i^{*}\right) \\
P_{t+1}=p_{t} i\left[1-f\left(p_{t}, i, P_{t} i^{*}\right)\right]
\end{array}
$$

onde $i^{*}$ representa a probabilidade de um adulto sobreviver à aplicação de inseticida. Para este caso, a função de sobrevivência do hospedeiro é escrita como

$$
f\left(p_{t}, i, p_{t}, i^{*}\right)=\left[1+\frac{a P_{t} i^{*}}{k\left(1+a p_{t} i\right)}\right]^{-k}
$$

As recorrências para os quatro tipos de efeitos de aplicação de inseticidas podem ser vistas na Figura 5. 
A

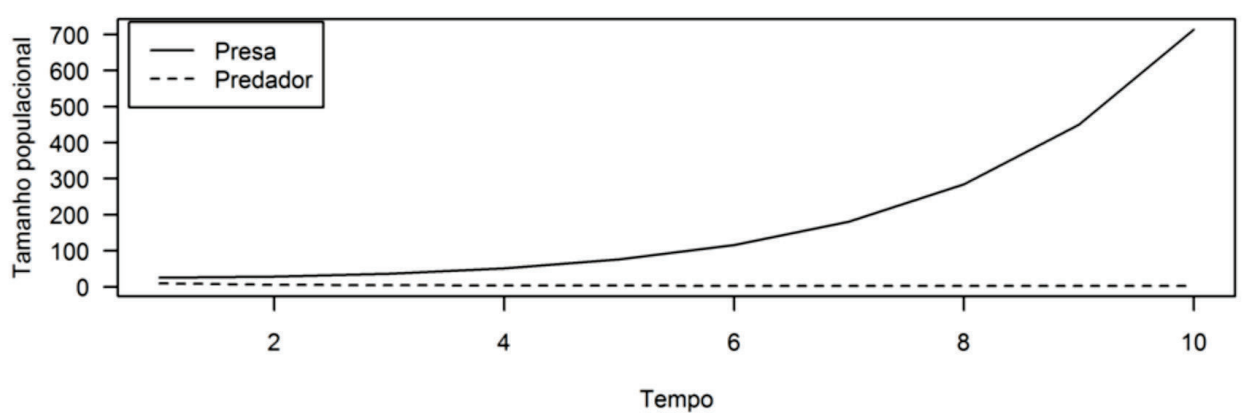

B

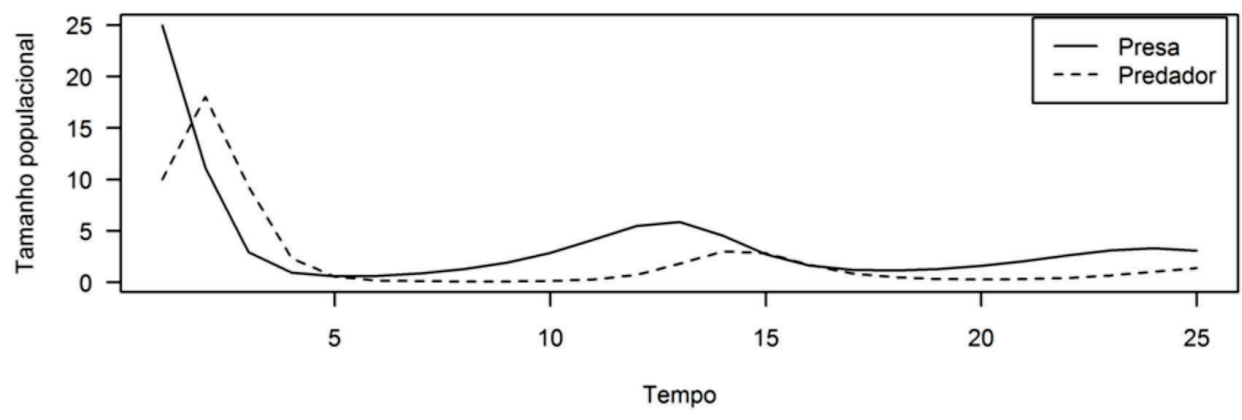

C

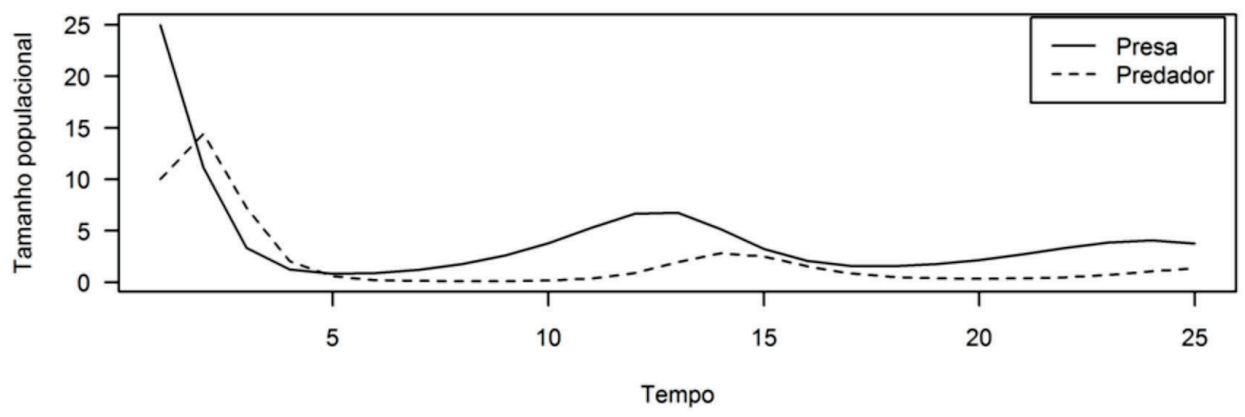

D

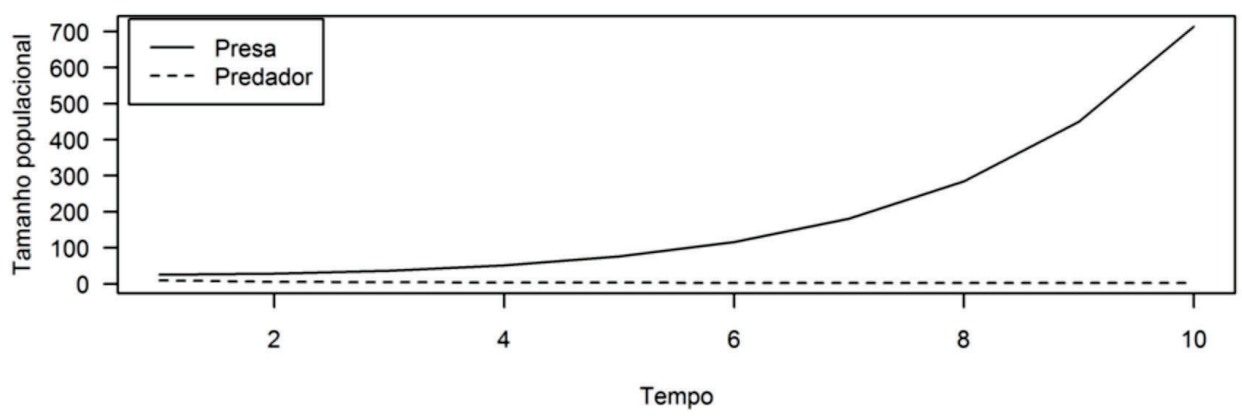

Figura 5. Recorrência no tempo para a dinâmica predador-presa com efeitos da aplicação de inseticidas. Valores dos parâmetros comuns a todos os casos: $r=2, a=1, k=0.6, i=0.8, p=25, P=10$. Antes da liberação de parasitóides resultando na eliminação somente de hospedeiros (A), $\theta=1 ;$ após o parasitismo e eliminando somente hospedeiros (B), $T_{m}=0.05$; após o parasitismo, porém eliminando também os hospedeiros parasitados $(\mathrm{C}), T_{m}=0.05$ e antes do parasitismo, porém eliminando também os parasitóides adultos (D), $i=1$.

Figure 5. Recurrence plot for the time series generated by the predator-prey dynamics with insecticide application effects. Parameter values common to every case: $r=2, a=1, k=0.6, i=0.8, p=25, P=10$. Before parasitoid release, resulting in elimination of only hosts (A), $\theta=1$; after parasitism and eliminating only hosts (B), $T_{m}=0.05$; after parasitism, however also eliminating parasitized hosts $(C), T_{m}=0.05$ and before parasitism, however also eliminating adult parasitoids $(D), i^{\prime}=1$. 
O parâmetro $i$ foi analisado no que diz respeito à sua sensibilidade às mudanças na sua magnitude. A análise foi feita empregando-se a teoria de bifurcação (May \& Oster 1976). O diagrama de bifurcação dá uma descrição global do espaço paramétrico, mostrando em um único gráfico, todos os comportamentos possíveis do sistema, para todos os valores do parâmetro.

Nota-se um crescimento irrestrito por parte do predador e da presa em determinada faixa do espectro paramétrico para as possibilidades 1 e 4 (Figura 6a, 6d). Isso é devido à formulação original do modelo de Hassell (1984), que não possui dependência da densidade. Assim, mesmo com a ação do inseticida antes do parasitismo a população cresce ilimitadamente. Algebricamente, isso significa que quando o tamanho populacional das presas tende ao infinito, tem-se:

$\lim _{p_{t} \rightarrow \infty} f\left(p_{t}, i, P_{t}\right)=\lim _{p_{t} \rightarrow \infty}\left[1+\frac{a P_{t}}{k\left(1+a \theta p_{t} i\right)}\right]^{-k}=1 \mathrm{e}$

A

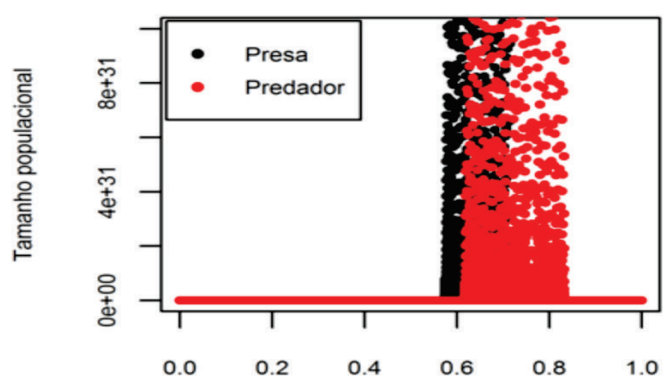

Sobrevivência influenciada pelo pesticida (i)

C

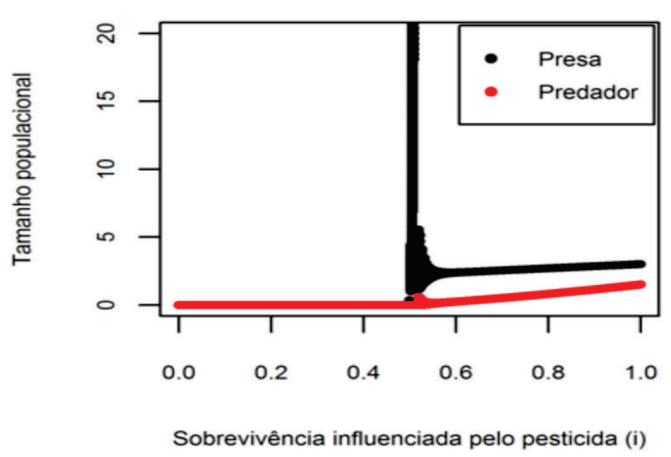

$\lim _{p_{t} \rightarrow \infty} f\left(p_{t}, i, P_{t}, i^{*}\right)=\lim _{p_{t} \rightarrow \infty}\left[1+\frac{a P_{t} i^{*}}{k\left(1+a p_{t} i\right)}\right]^{-k}=1$,

implicando, para o parasitóide, em extinção, pois

$$
\begin{aligned}
& \lim _{p_{t} \rightarrow \infty}\left[1-f\left(p_{t}, i, P_{t}\right)\right]=0 e \\
& \lim _{p_{t} \rightarrow \infty}\left[1-f\left(p_{t}, i, P_{t}, i^{*}\right)\right]=0 .
\end{aligned}
$$

Assim, sendo o parasitóide o único fator limitante para o sistema e considerando que parte da população de hospedeiros, apesar de atingida pelo inseticida, não sofre mortalidade integral, observa-se que o crescimento da população de praga (hospedeiro) não tem contenção. Contudo, as formulações propostas não caracterizam a dinâmica de um sistema real, pois, conforme hospedeiros aumentam infinitamente, parasitóides são extintos. O evento esperado seria o crescimento dos hospedeiros sendo acompanhado pelo parasitóide, por tratar-se do recurso exclusivo e necessário à subsistência populacional, como se vê nas possibilidades descritas pelas Figuras $5 \mathrm{~b}, \mathrm{c}, 6 \mathrm{~b}$ e c.

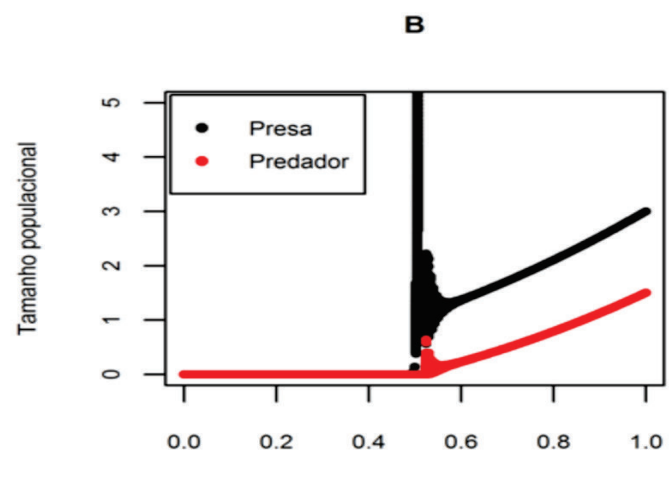

Sobrevivência influenciada pelo pesticida (i)

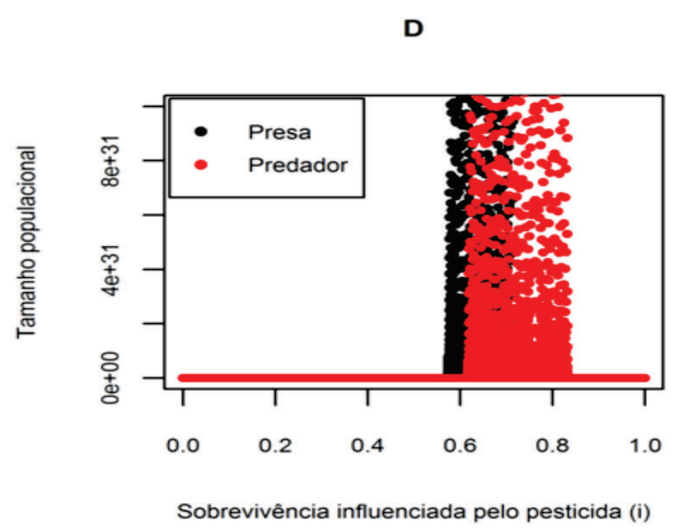

Figura 6. Diagramas de bifurcação para análise da relação entre tamanho populacional e sobrevivência influenciada pelo inseticida com presas exibindo crescimento irrestrito (A, D). Valores dos parâmetros são os mesmos dos utilizados na Figura 5.

Figure 6. Bifurcation diagrams for the analysis of the relationship between population size and survival influenced by the insecticide with prey exhibiting unrestricted growth $(A, D)$. The parameter values are the same used in Figure 5. 


\section{INTRODUZINDO DEPENDENNCIA DA DENSIDADE}

Ao investigar a dinâmica com $i=0.52$ (Figura 7), nota-se que a amplificação do espectro aparece na região de transiência que se estende por mais de 700 gerações. Isso, no contexto de manejo de pragas deve ser considerado importante no tocante ao planejamento de estratégias de controle (Kidd \& Amarasekare 2011). No início da implantação do controle, pode haver diferentes tipos de comportamento dinâmico, todos importantes para que a estratégia implementada seja reavaliada e, posteriormente, reestruturada.

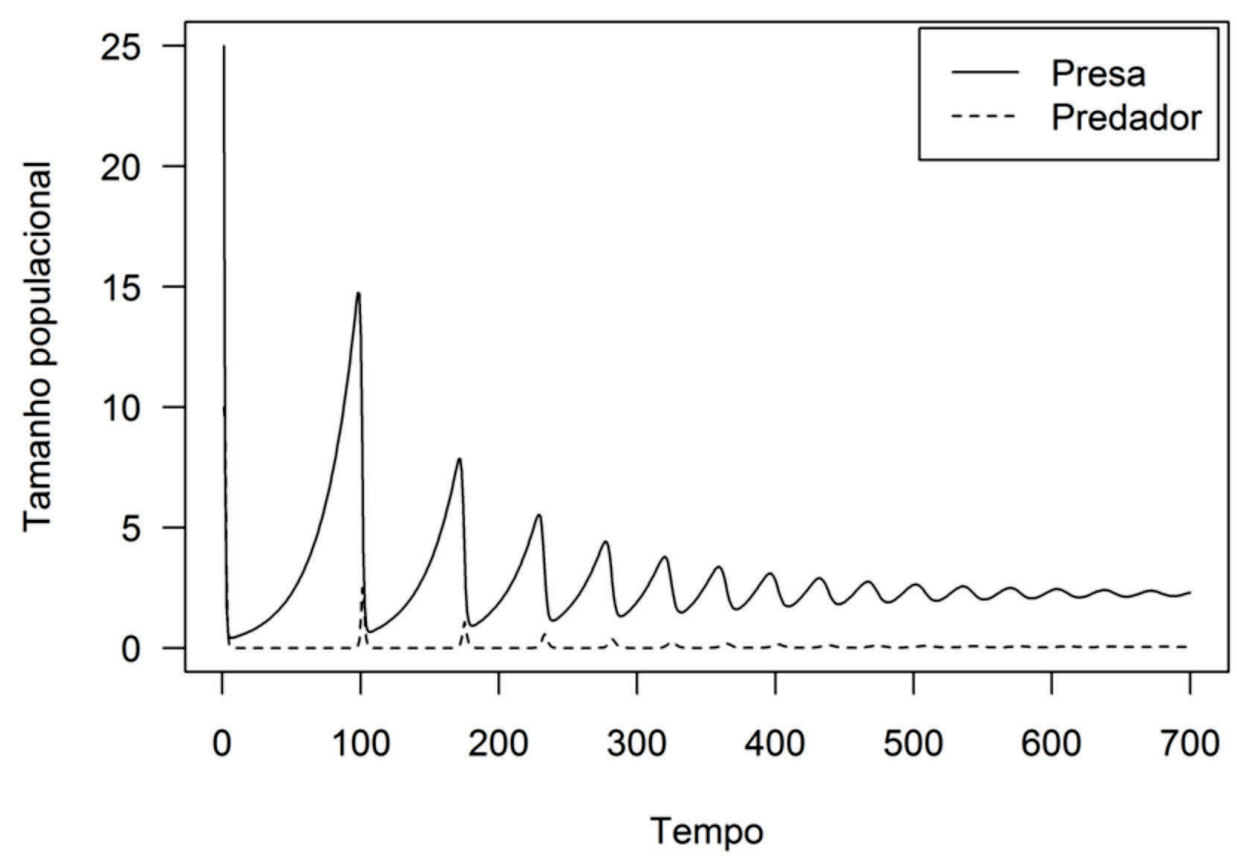

Figura 7. Dinâmica transiente indicando comportamento assintótico tendendo à estabilidade para presa e predador. Parâmetros: $r=2$, $a=1, k=0.6, T_{m}=0.05, i=0.52, p=25, P=10$.

Figure 7. Transient dynamics indicating asymptotic behavior which tends towards stability for the prey and the predator. Parameter values: $r=2, a=1, k=0.6, T_{m}=0.05, i=0.52, p=25, P=10$.

Para corrigir o efeito resultante da explosão populacional de presas nos modelos propostos por Hassel (1984), a dependência da densidade pode ser inserida, sob forma do parâmetro $K$, que representa a capacidade de suporte do agroecossistema. Assim, os modelos são escritos da seguinte forma:

- Possibilidade 1:

$$
\begin{gathered}
p_{t+1}=r p_{t}\left(1-\frac{p_{t}}{K}\right) i f\left(p_{t}, i, P_{t}\right) \\
P_{t+1}=p_{t} i\left[1-f\left(p_{t}, i, P_{t}\right)\right]
\end{gathered}
$$

- Possibilidade 2:

$$
\begin{gathered}
p_{t+1}=r p_{t}\left(1-\frac{p_{t}}{K}\right) f\left(p_{t}, P_{t}\right) i \\
P_{t+1}=p_{t}\left[1-f\left(p_{t}, P_{t}\right)\right]
\end{gathered}
$$

- Possibilidade 3:

$$
\begin{gathered}
p_{t+1}=r p_{t}\left(1-\frac{p_{t}}{K}\right) f\left(p_{t}, P_{t}\right) i \\
P_{t+1}=p_{t} i\left[1-f\left(p_{t}, P_{t}\right)\right]
\end{gathered}
$$

- Possibilidade 4:

$$
\begin{gathered}
p_{t+1}=r p_{t}\left(1-\frac{p_{t}}{K}\right) i f\left(p_{t}, i, P_{t}, i^{*}\right) \\
P_{t+1}=p_{t} i\left[1-f\left(p_{t}, i, P_{t}, i^{*}\right)\right]
\end{gathered}
$$

As dinâmicas simuladas para as equações 31 à 38 mostram que a inserção da capacidade suporte gera estabilidade maior no sistema (Figura 8), contornando o problema decorrente do crescimento ilimitado de presas. 
A

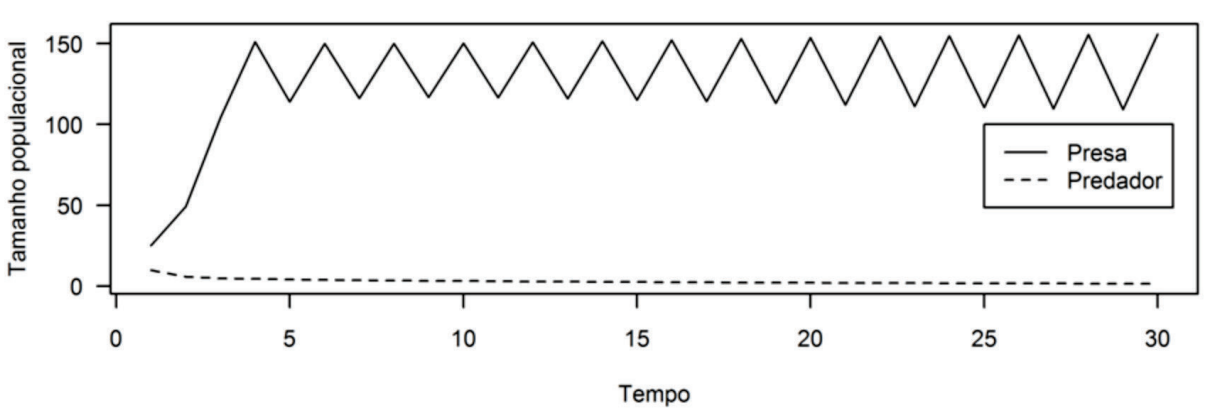

B

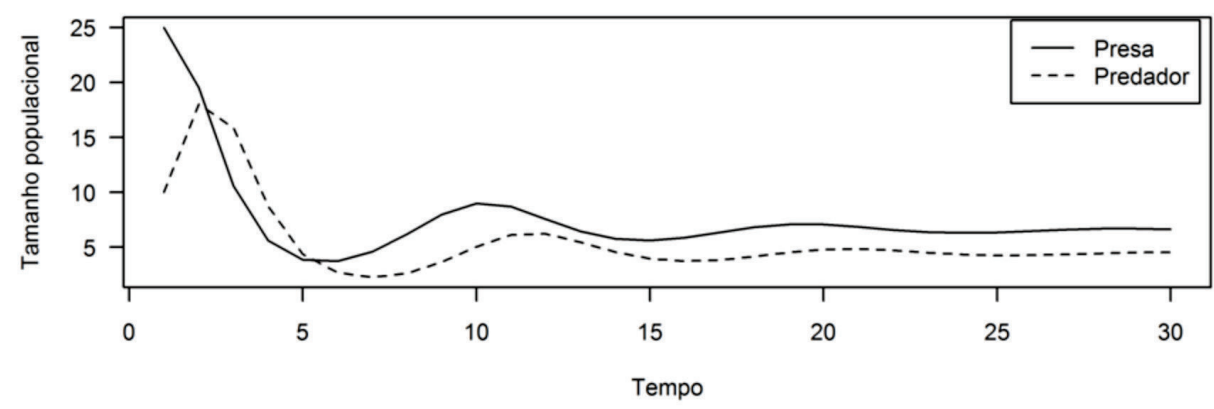

C

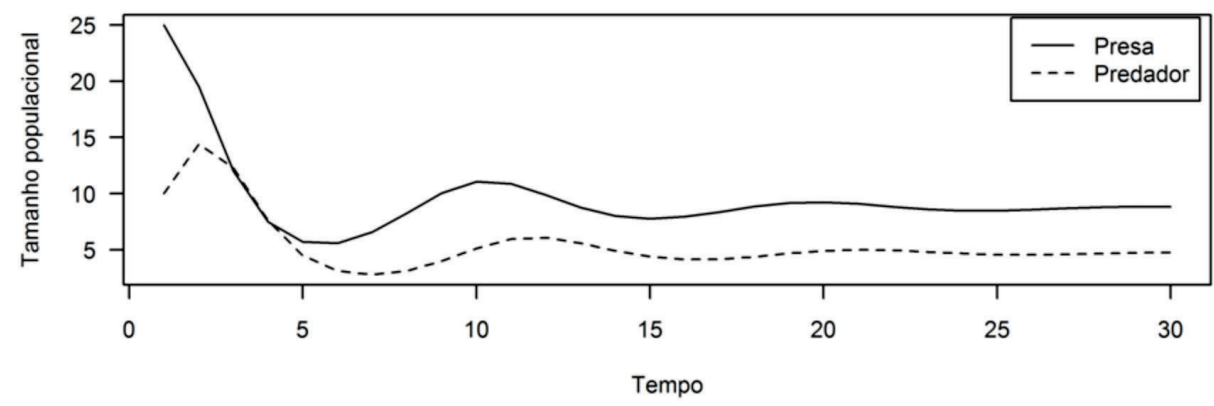

D

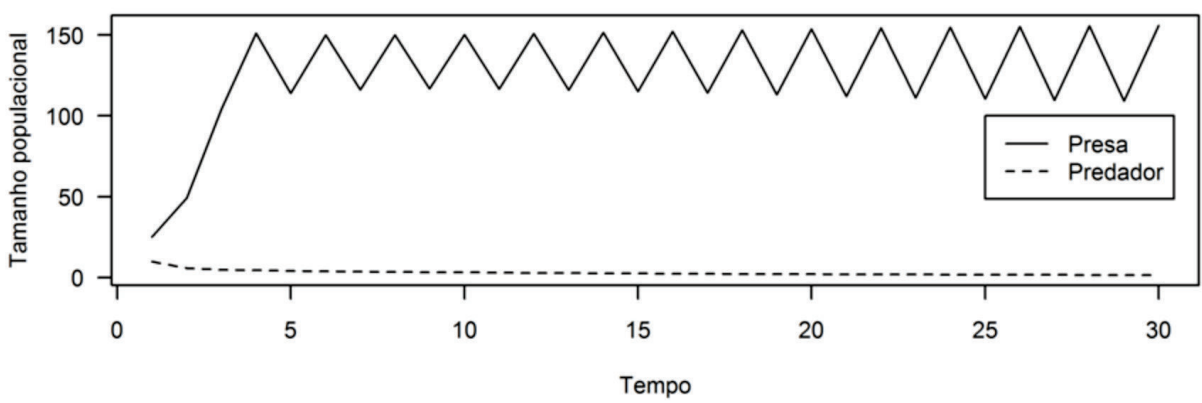

Figura 8. Recorrência no tempo para a dinâmica predador-presa incluindo dependência da densidade e efeitos da aplicação de inseticidas antes da liberação de parasitóides resultando na eliminação somente de hospedeiros (A), após o parasitismo e eliminando somente hospedeiros (B), após o parasitismo, porém eliminando também os hospedeiros parasitados (C) e antes do parasitismo, porém eliminando também os parasitóides adultos (D). Valores dos parâmetros idênticos aos da Figura 5, exceto para $r=4$. Nesta simulação foi incluído o parâmetro $K=200$, correspondente à capacidade suporte.

Figure 8. Recurrence plots for the time series generated by the predator-prey dynamics including density dependence and insecticide application effects before parasitoid liberation, resulting in the elimination of only hosts (A), after parasitism and eliminating only hosts (B), after parasitism, however also eliminating parasitized hosts $(C)$ and before parasitism, however also eliminating adult parasitoids (D). Parameter values identical to the ones used in Figure 5, except for $r=4$. In this simulation, the parameter $K=200$ was included, which corresponds to the carrying capacity. 
A análise de sensibilidade do parâmetro $i$ revela equilíbrio estável para a espécie predadora e comportamento dinâmico caracterizado pela perda da estabilidade com a diminuição da magnitude do efeito do inseticida ( $i$ se aproxima de 1). O resultado do efeito pode ser visto no diagrama de bifurcação (Figuras 9a, d). Com alta influência do efeito do inseticida sobre a sobrevivência populacional, o resultado observado é o equilíbrio estável de um ponto. Com o aumento do valor do parâmetro, há uma duplicação de período, resultando em ciclo limite de dois pontos. Novas duplicações de período ocorrem em função dos valores superiores de $i$, resultando em comportamento caótico (Figuras 9a, d). Nota-se que a transiência também não foi completamente eliminada para produzir os diagramas das Figuras $9 \mathrm{~b}$ e $9 \mathrm{c}$. Para as possibilidades 2 e 3 (Figuras 9b, c), os diagramas de bifurcação indicam um equilíbrio estável de um ponto em quase todo o espectro de valores atribuídos ao parâmetro $i$.
A

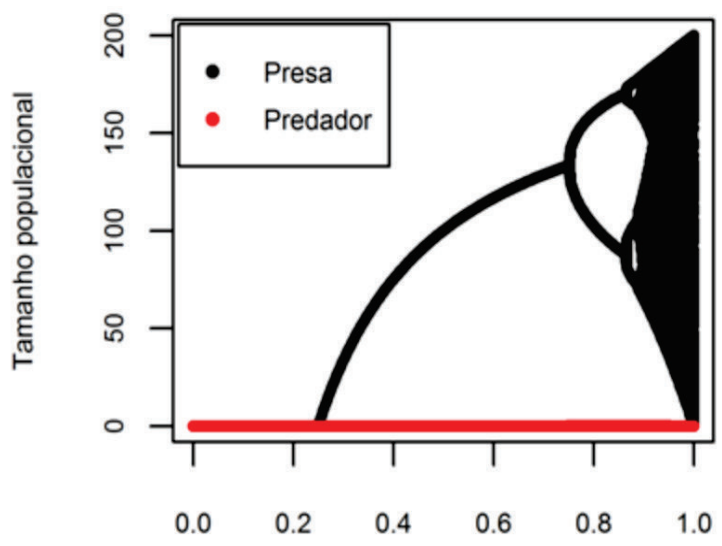

Sobrevivência influenciada pelo pesticida (i)

C

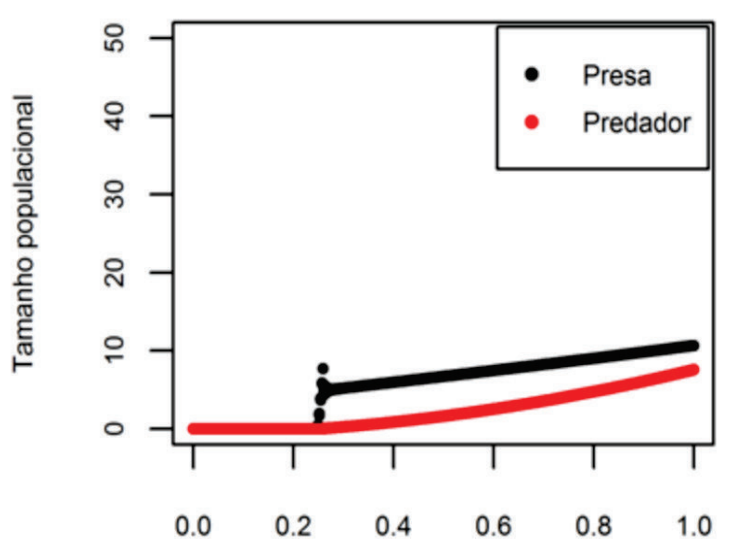

Sobrevivência influenciada pelo pesticida (i)
B

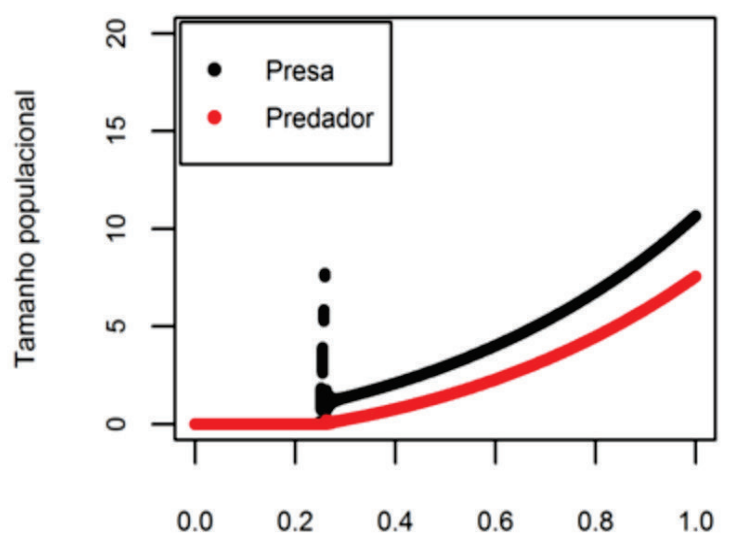

Sobrevivência influenciada pelo pesticida (i)

D

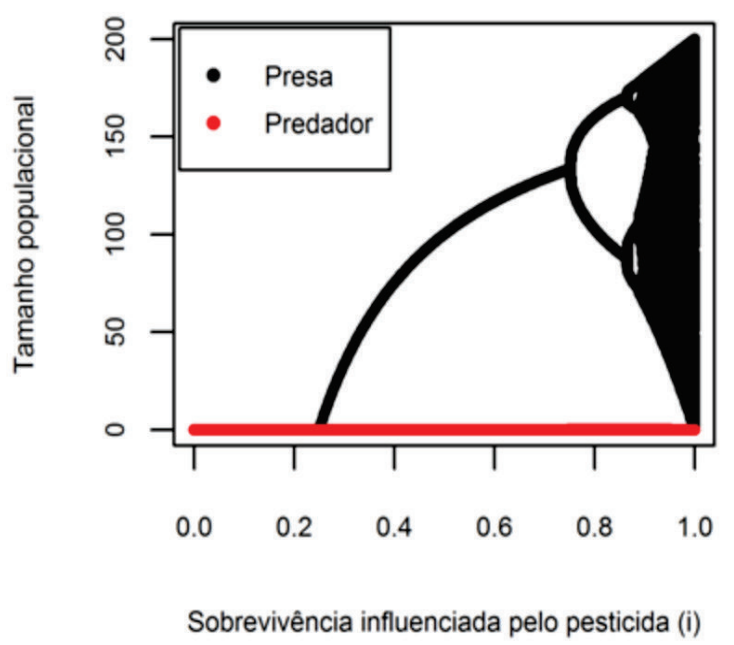

Figura 9. Diagramas de bifurcação para a análise de sensibilidade da sobrevivência influenciada pelo inseticida (i). Valores dos parâmetros idênticos aos valores da Figura 8.

Figure 9. Bifurcation diagrams for the analysis of sensitivity of the survival influenced by the insecticide (i). Parameter values identical to those of Figure 8. 


\section{INTRODUZINDO ESTOCASTICIDADE}

Os modelos até agora apresentados são caracterizados como determinísticos, isto é, todos os parâmetros são fixos durante todos os passos de tempo. É possível introduzir um efeito estocástico em um ou mais parâmetros, fazendo-os variar ao longo do tempo segundo alguma distribuição estatística (Serra et al. 2007). Isso retrataria efeitos do acaso ou perturbações ao longo do tempo que podem acontecer no sistema. Ao deixar os parâmetros referentes à sobrevivência influenciada pelo inseticida variarem segundo uma distribuição normal, obtêm-se os seguintes modelos:

- Possibilidade 1:

$$
\begin{aligned}
& p_{t+1}=r p_{t}\left(1-\frac{p_{t}}{K}\right) i f\left(p_{t}, i, P_{t}\right), i \sim N\left(\mu, \sigma^{2}\right) \\
& P_{t+1}=p_{t} i\left[1-f\left(p_{t}, i, P_{t}\right)\right], i \sim N\left(\mu, \sigma^{2}\right)
\end{aligned}
$$

- Possibilidade 2:

$$
\begin{gathered}
p_{t+1}=r p_{t}\left(1-\frac{p_{t}}{K}\right) f\left(p_{t}, P_{t}\right) i, i \sim N\left(\mu, \sigma^{2}\right) \\
P_{t+1}=p_{t}\left[1-f\left(p_{t}, P_{t}\right)\right], i \sim N\left(\mu, \sigma^{2}\right)
\end{gathered}
$$

- Possibilidade 3:

$$
\begin{gathered}
p_{t+1}=r p_{t}\left(1-\frac{p_{t}}{K}\right) f\left(p_{t}, P_{t}\right) i, i \sim N\left(\mu, \sigma^{2}\right) \\
P_{t+1}=p_{t} i\left[1-f\left(p_{t}, P_{t}\right)\right], i \sim N\left(\mu, \sigma^{2}\right)
\end{gathered}
$$

- Possibilidade 4:

$$
\begin{gathered}
p_{t+1}=r p_{t}\left(1-\frac{p_{t}}{K}\right) i f\left(p_{t}, i, P_{t}, i^{*}\right), i \sim N\left(\mu_{i}, \sigma_{i}\right), i^{*} \sim N\left(\mu_{i^{*}}, \sigma^{2} i^{*}\right) \\
P_{t+1}=p_{t} i\left[1-f\left(p_{t}, i, P_{t}, i^{*}\right)\right], i \sim N\left(\mu_{i}, \sigma_{i}\right), i^{*} \sim N\left(\mu_{i^{*}}, \sigma^{2}{ }_{i}^{*}\right)
\end{gathered}
$$

Limitando os possíveis valores sorteados observando-se as dinâmicas apresentadas na para $i$ e $i^{*}$, segundo a distribuição normal, Figura 10, nota-se que a dinâmica predador-presa entre 0 e 1, pode-se simular os efeitos de uma é caracterizada por oscilações instáveis advindas eficiência instável do inseticida aplicado. Assim, da estocasticidade. 
A

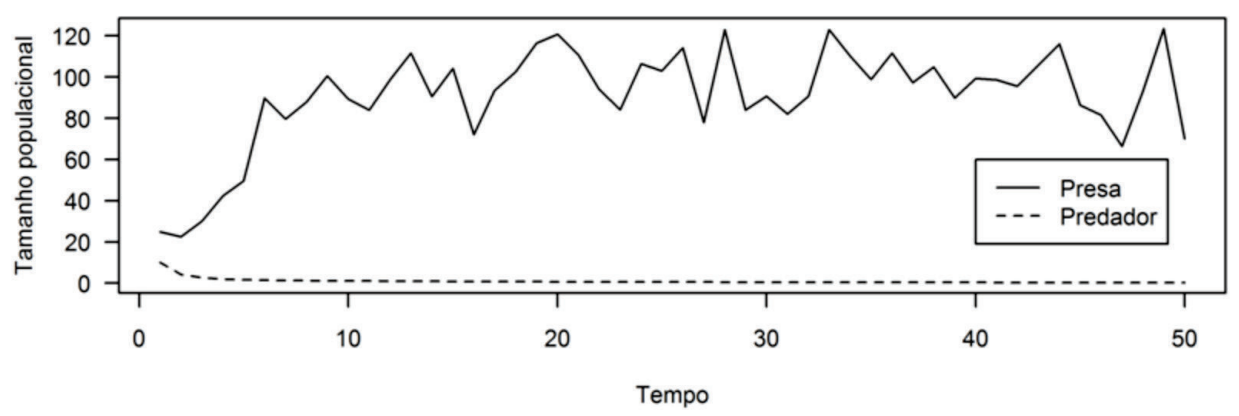

B

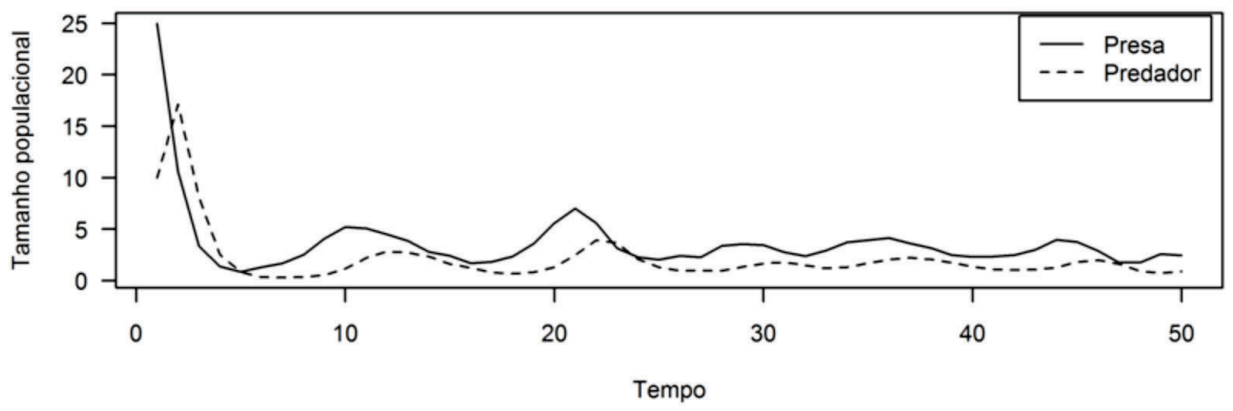

C

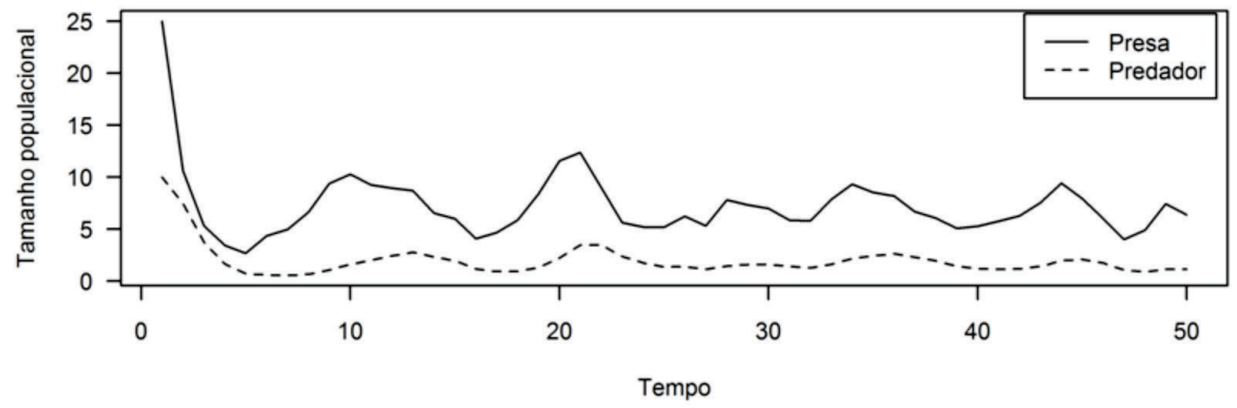

D

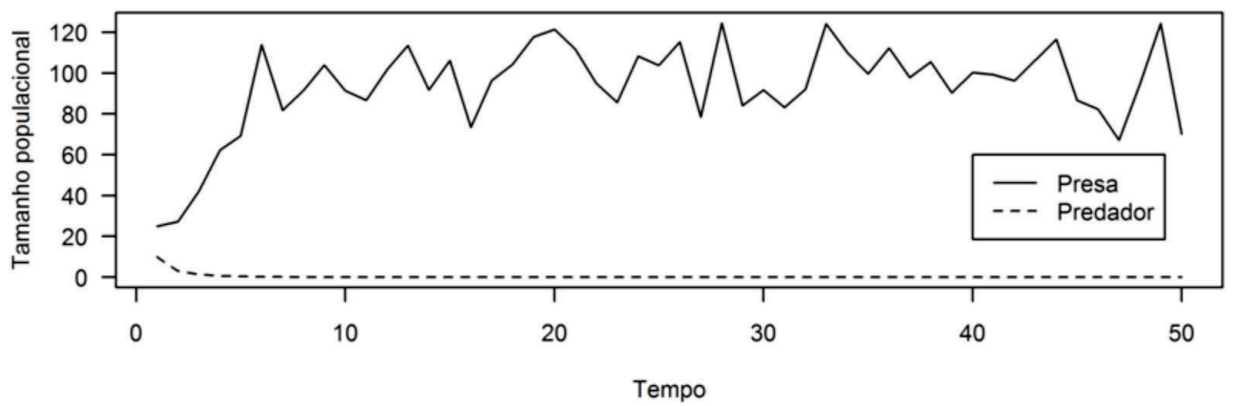

Figura 10. Recorrência no tempo para a dinâmica predador-presa incluindo dependência da densidade, estocasticidade, efeitos da aplicação de inseticidas antes da liberação de parasitóides resultando na eliminação somente de hospedeiros (A), após o parasitismo e eliminando somente hospedeiros (B), após o parasitismo, porém eliminando também os hospedeiros parasitados (C) e antes do parasitismo, porém eliminando também os parasitóides adultos (D). Valores dos parâmetros idênticos à Figura 8. Estocasticidade em $i$ e $i$, seguindo distribuição normal com média igual à 0.5 e variância igual à 0.01 . Figure 10. Recurrence plots for the time series generated by the predator-prey dynamics including density dependence, stochasticity, insecticide application effects before parasitoid liberation, resulting in the elimination of only hosts (A), after parasitism and eliminating only hosts (B), after parasitism, however also eliminating parasitized hosts $(C)$ and before parasitism, however also eliminating adult parasitoids (D). Parameter values identical to those of Figure 8. Stochasticity in $i$ and $i^{\prime}$, which are normally distributed with mean equal to 0.5 and variance equal to 0.01 .. 
INTRODUZINDO PERIODICIDADE DE APLICAÇÃO DO INSETICIDA

Diversos estudos utilizaram funções para introduzir efeitos periódicos em modelos (Banks et al. 2008, Zhang et al. 2008). Supondo um cenário de aplicação periódica de inseticida, o efeito residual resultante da aplicação diminui até o momento da próxima aplicação. Um caso, no qual o inseticida é aplicado a cada 20 dias com efeito residual variando de 1 (aplicação) até 0,5 (Figura 11), pode ser modelado com uma função trigonométrica:

$$
I(t)=0,75+0,25 \cos \left(\frac{t \pi}{10}\right)
$$

A função é introduzida no modelo no lugar do parâmetro $i$, de modo a gerar os modelos:

- Possibilidade 1:

$$
\begin{aligned}
& p_{t+1}=r p_{t}\left(1-\frac{p_{t}}{K}\right) I(t) f\left(p_{t}, I(t), P_{t}\right) \\
& P_{t+1}=p_{t} I(t)\left[1-f\left(p_{t}, I(t), P_{t}\right)\right]
\end{aligned}
$$

- Possibilidade 2:

$$
\begin{gathered}
p_{t+1}=r p_{t}\left(1-\frac{p_{t}}{K}\right) f\left(p_{t}, P_{t}\right) I(t) \\
P_{t+1}=p_{t}\left[1-f\left(p_{t}, P_{t}\right)\right]
\end{gathered}
$$

- Possibilidade 3:

$$
\begin{aligned}
& p_{t+1}=r p_{t}\left(1-\frac{p_{t}}{K}\right) f\left(p_{t}, P_{t}\right) I(t) \\
& P_{t+1}=p_{t} I(t)\left[1-f\left(p_{t}, P_{t}\right)\right]
\end{aligned}
$$

- Possibilidade 4:

$$
\begin{gathered}
p_{t+1}=r p_{t}\left(1-\frac{p_{t}}{K}\right) I(t) f\left(p_{t}, I(t), P_{t}, i^{*}\right) \\
P_{t+1}=p_{t} I(t)\left[1-f\left(p_{t}, I(t), P_{t}, i^{*}\right)\right]
\end{gathered}
$$

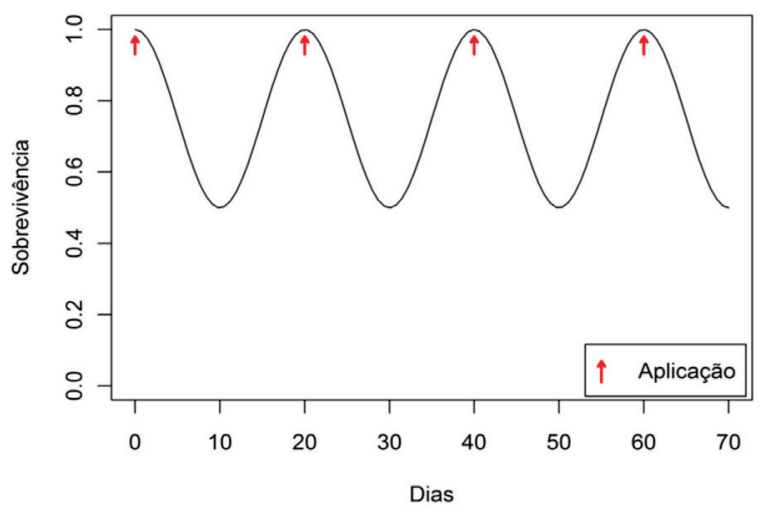

Figura 11. Periodicidade de aplicação de inseticidas no sistema. Figure 11. Periodicity of insecticide application in the system.

A Figura 12 (a,b,c,d) descreve a dinâmica do sistema predador-presa incluindo a periodicidade de aplicação de inseticida em modelo com capacidade suporte. Das quatro possibilidades analisadas as Figuras $12 \mathrm{~b}$ e $12 \mathrm{c}$ descrevem as melhores trajetórias para predador e presa. Isto reflete oscilações recorrentes situando-se em tamanhos populacionais de baixa magnitude, porém indicando sustentabilidade ao sistema, o que caracteriza comportamento dinâmico representado por ciclos periódicos estáveis.

A esses modelos pode ser introduzida estocasticidade no efeito do inseticida, a partir da reintrodução do parâmetro $i$, distribuído normalmente ao longo do tempo:

- Possibilidade 1:

$p_{t+1}=r p_{t}\left(1-\frac{p_{t}}{K}\right) I(t) i f\left(p_{t}, I(t), i, P_{t}\right), i \sim N\left(\mu, \sigma^{2}\right)$

$$
P_{t+1}=p_{t}\left(1-\frac{P_{t}}{K}\right) I(t) i\left[1-f\left(p_{t}, I(t), i, P_{t}\right)\right], i \sim N\left(\mu, \sigma^{2}\right)
$$

- Possibilidade 2:

$$
\begin{gathered}
p_{t+1}=r p_{t}\left(1-\frac{p_{t}}{K}\right) f\left(p_{t}, P_{t}\right) I(t) i, i \sim N\left(\mu, \sigma^{2}\right) \\
P_{t+1}=p_{t}\left(1-\frac{P_{t}}{K}\right)\left[1-f\left(p_{t}, P_{t}\right)\right], i \sim N\left(\mu, \sigma^{2}\right)
\end{gathered}
$$

- Possibilidade 3:

$$
\begin{aligned}
& p_{t+1}=r p_{t}\left(1-\frac{p_{t}}{K}\right) f\left(p_{t}, P_{t}\right) I(t) i, i \sim N\left(\mu, \sigma^{2}\right) \\
& p_{t+1}=r p_{t}\left(1-\frac{p_{t}}{K}\right) f\left(p_{t}, P_{t}\right) I(t) i, i \sim N\left(\mu, \sigma^{2}\right)
\end{aligned}
$$


- Possibilidade 4:

$$
\begin{gathered}
p_{t+1}=r p_{t}\left(1-\frac{p_{t}}{K}\right) I(t) i f\left(p_{t}, I(t), i, P_{t}, i^{*}\right), i \sim N\left(\mu_{i}, \sigma_{i}\right), i^{*} \sim N\left(\mu_{i^{*}}, \sigma^{2} i^{*}\right) \\
P_{t+1}=p_{t} I(t) i\left[1-f\left(p_{t}, I(t), i, P_{t}, i^{*}\right)\right], i \sim N\left(\mu_{i}, \sigma_{i}\right), i^{*} \sim N\left(\mu_{i^{*}}, \sigma^{2} i_{i^{*}}\right) \\
f\left(p_{t}, I(t), i, P_{t}\right)=\left[1+\frac{a P_{t}}{k\left(1+a \theta p_{t} I(t) i\right)}\right]^{-k}
\end{gathered}
$$

com

$$
f\left(p_{t}, I(t), i, p_{t}, i^{*}\right)=\left[1+\frac{a P_{t} i^{*}}{k\left(1+a p_{t} I(t) i\right)}\right]^{-k}
$$

A

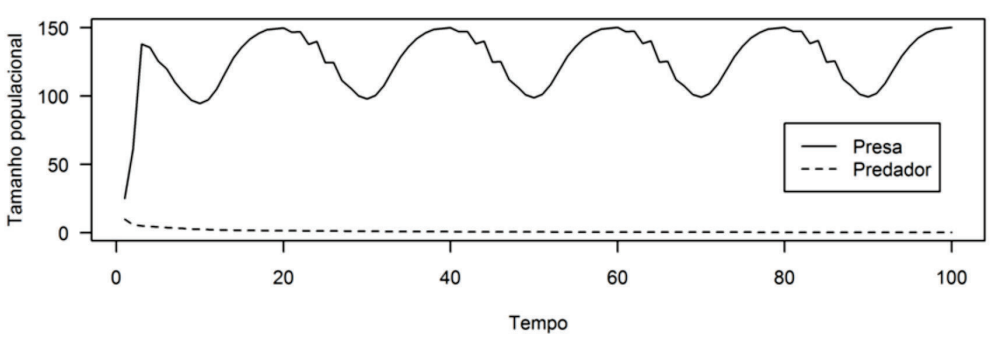

B

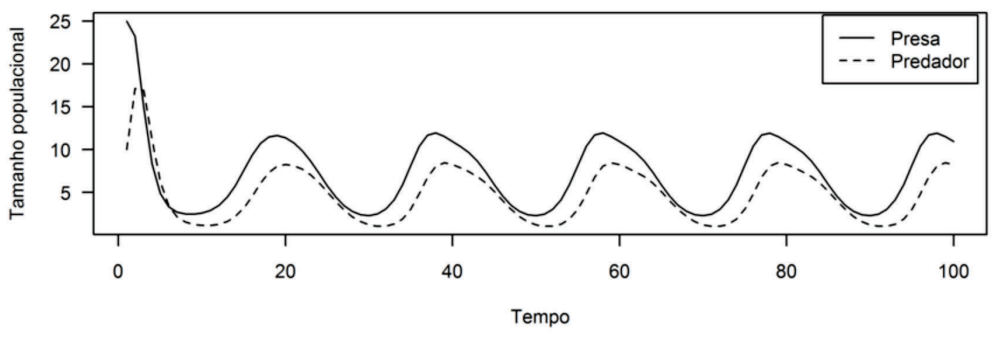

C

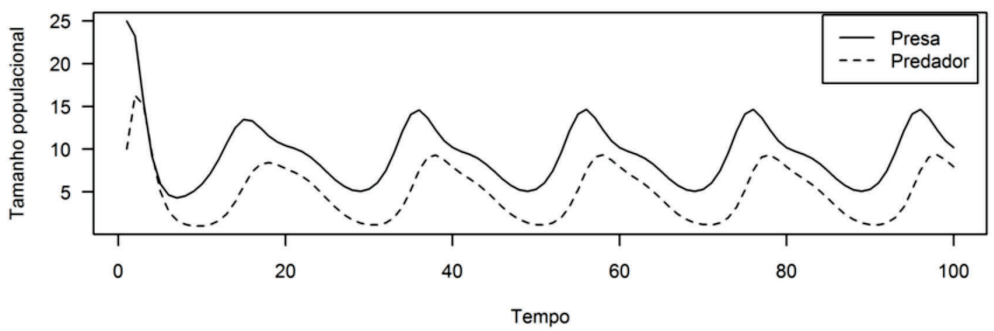

D

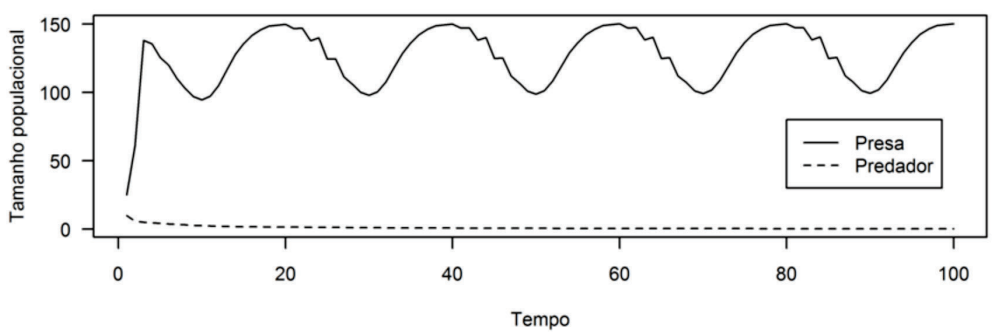

Figura 12. Dinâmica do sistema predador-presa incluindo a periodicidade de aplicação de inseticida em modelo com capacidade suporte inserida. Valores dos parâmetros idênticos à Figura 8.

Figure 12. Dynamics of the predator-prey system including periodicity of insecticide application in a model with included carrying capacity. Parameter values identical to those of Figure 8. 
Na Figura 13 (a,b,c,d) nota-se a dinâmica predador-presa com efeito periódico da ação do inseticida sujeito à estocasticidade, segundo distribuição normal. É interessante notar a similaridade entre as possibilidades 1 e 4 em todas as simulações, bem como a similaridade entre as possibilidades 2 e 3. Isso é devido ao parâmetro $i$ inserido dentro do termo da resposta funcional do predador apenas nas possibilidades 1 e 4 . Isso implica em uma menor densidade de presas disponíveis para o predador, resultando em densidade menor de predadores ao longo do tempo. Nas possibilidades 2 e 3, o predador tem toda a densidade de presas disponível no termo de resposta funcional, resultando em uma dinâmica na qual a densidade de predadores acompanha as presas mais nitidamente.

A

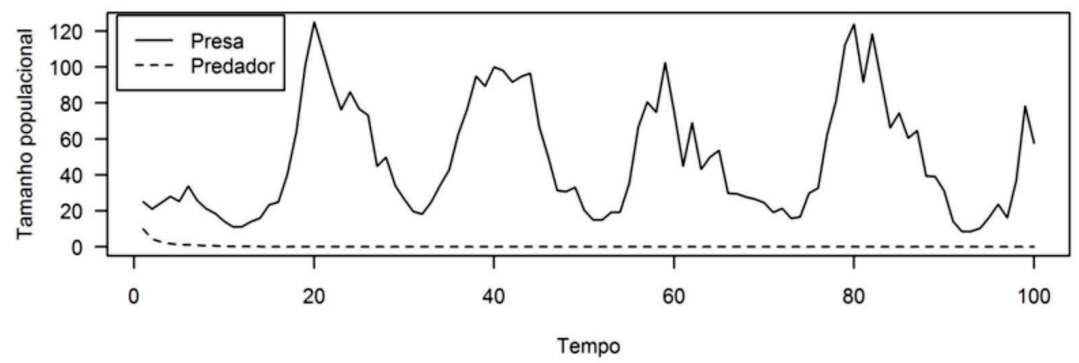

B

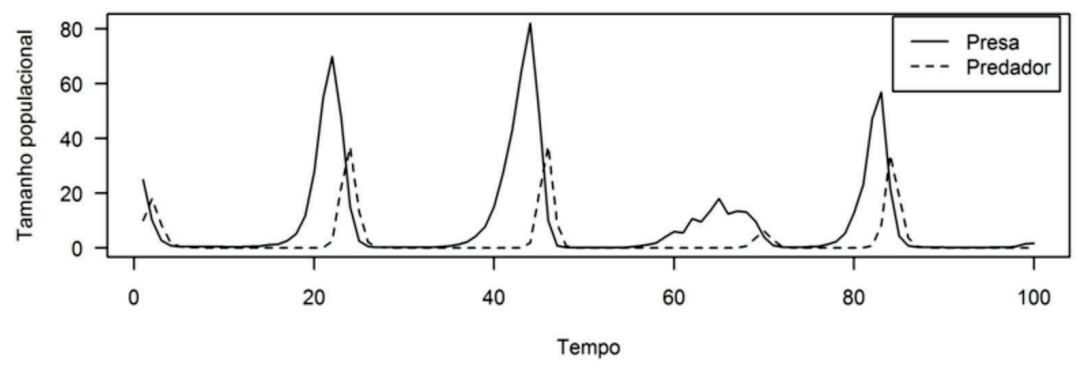

C

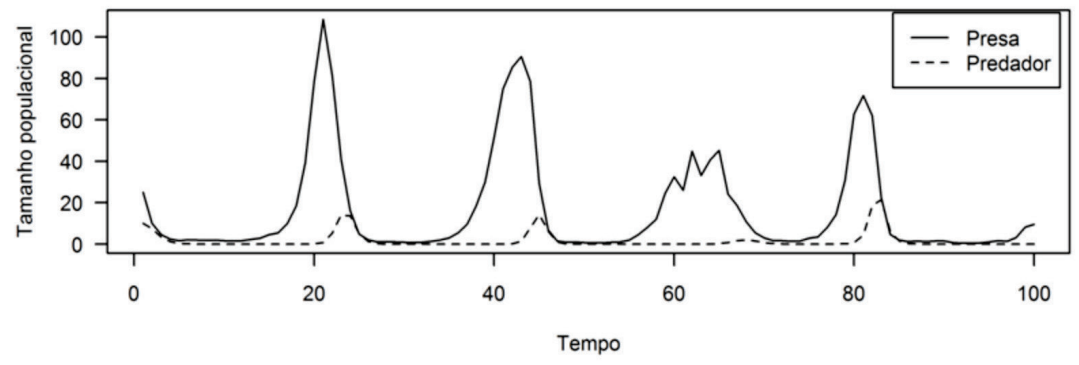

D

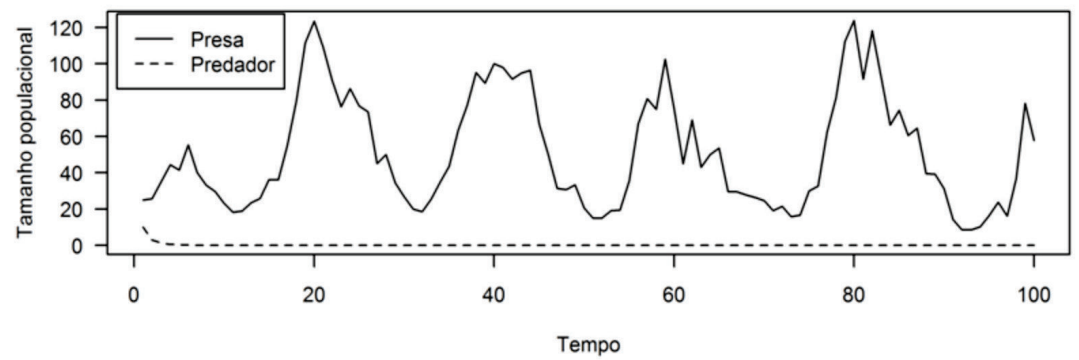

Figura 13. Dinâmica predador-presa com efeito periódico da ação do inseticida sujeito à estocasticidade, segundo distribuição normal. Valores dos parâmetros idênticos à Figura 10.

Figure 13. Predator-prey dynamics with stochastic periodic effect of the insecticide action, normally distributed. Parameter values identical to those of Figure 10. 


\section{INCORPORANDO DIFERENTES ESTRATÉGIAS}

A despeito do estudo de Hassell (1984) ter representado ótima oportunidade para investigar a combinação entre diferentes estratégias de controle de pragas no contexto da ecologia teórica, do ponto de vista prático, nenhum estudo com modelos ecológicos realizado nas últimas décadas incorporou estratégias de manejo de pragas como fizeram recentemente Zang et al. (2008) e Tang \& Cheke (2008). O modelo proposto por Tang \& Cheke (2008) leva em conta as bases ecológicas apresentadas no presente estudo, presentes através da descrição contida nas equações (1) à (19). Tang \& Cheke (2008) incorporaram o conceito de manejo integrado às equações de Nicholson \& Bailey (1935), considerando a política de monitoramento periódico de pragas e levando em conta a determinação do limiar e do nível de dano econômico (Pedigo \& Zeiss 1996, Lima et al. 2010).

A idéia original de Tang \& Cheke (2008) foi desenvolvida a partir de equações diferenciais similares às equações (1) e (2) que aparecem no presente estudo. $\mathrm{O}$ uso de equações diferenciais para descrever a dinâmica hospedeiro-parasitóide em tempo contínuo foi proposto por estes autores para casos em que o estudo da dinâmica prevê a análise tanto dentro como entre gerações. Além disso, para alguns sistemas, as gerações de hospedeiros podem exibir sobreposição. As equações de Tang \& Cheke (2008) descrevem, assim, o sistema hospedeiroparasitóide que, no presente texto, para facilitar o entendimento, são dadas por notação similar à utilizada previamente (Equações 1 e 2):

$$
\begin{gathered}
\frac{d p}{d t}=r p-f(p, P) P \\
\frac{d P}{d t}=n f(p, P) P-m P
\end{gathered}
$$

Para introduzir a estratégia de manejo integrado no sistema, Tang \& Cheke (2008) consideraram que, caso o tamanho da população de pragas não atingisse o limiar de dano econômico (LD), o sistema hospedeiro-parasitóide seria governado apenas pelo formalismo teórico proposto nas equações (66) e (67). Do contrário, as populações seriam governadas pela extensão do modelo descrito pelas equações acima, que pode ser escrita como:

$$
\begin{gathered}
p^{t^{+}}=\left(1-z_{1}\right) p_{t} \\
P^{t^{+}}=\left(1-z_{2}\right) P_{t}+\beta \\
p\left(0^{+}\right)=p_{0}<L D, P\left(0^{+}\right)=P_{0} .
\end{gathered}
$$

Nas equações acima, $p^{t^{+}} \mathrm{e} \quad P^{t^{+}}$representam o número de hospedeiros e parasitoides, respectivamente, após determinada estratégia de controle aplicada no tempo $t$ assim como, $p\left(0^{+}\right) e$ $P\left(0^{+}\right)$as densidades iniciais de hospedeiros e parasitóides. O termo $z_{1}$ que aparece na equação (68) é a proporção na qual a densidade da praga é reduzida por algum método de controle que não envolva o uso de parasitóide ou predador, caso atinja o LD. A magnitude do termo deve ser maior ou igual à zero e menor que 1. O termo $z_{2}$ que aparece na equação (69) determina uma proporção para a taxa de liberação de parasitóides, com magnitude maior ou igual à zero. $\beta$ é o número constante de parasitóides liberados no tempo $t$. O modelo proposto por Tang \& Cheke (2008) foi revisitado por Lima et al. (2009), que propuseram uma extensão ao modelo, introduzindo a dimensão do espaço no sistema. Lima et al. (2009) demonstraram, numericamente, a diferença entre o modelo original proposto por Nicholson \& Bailey (1935), o formalismo teórico de Tang \& Cheke (2008) e a proposição do modelo com a estratégia MIP (Tang \& Cheke 2008) recebendo a estrutura espacial por meio de mapas acoplados em reticulados (Lima et al. 2009). Os resultados indicam que a introdução da estratégia MIP no modelo teórico permite a intervenção no tamanho populacional da espécie praga quando se estabelece um limiar de dano econômico. Além disso, o arcabouço teórico pertinente à dimensão de espaço introduzida no modelo de Tang \& Cheke (2009) revela que a estrutura espacial é essencial para modelos desta natureza, já que sua introdução no sistema altera significativamente os valores de nível de dano econômico (Lima et al. 2009).

\section{CONSIDERAÇÕES E PERSPECTIVAS PARA UM FUTURO PRÓXIMO}

Modelar sistemas tróficos não é uma tarefa trivial, sobretudo no contexto de manejo de pragas. 
A complexidade inerente dos agroecossistemas envolve questões multifatoriais que demandam muitas vezes análises pormenorizadas do sistema, a fim de estabelecer conduta de parametrização adequada, realçando os aspectos realmente importantes para o processo de modelagem. O desafio é então selecionar processos que descrevam, sucintamente, o sistema ao qual se pretende modelar, porém sem perder elementos básicos que o governam (Schaalje et al. 1989). O presente estudo serviu para apresentar e realçar as bases ecológicas essenciais para a modelagem da dinâmica populacional de pragas e inimigos naturais, valendo-se da teoria predador-presa direcionada ao manejo integrado de pragas. Os estudos realizados com formulações matemáticas aplicados ao contexto de controle de pragas nem sempre têm combinado diferentes estratégias para o manejo integrado (Banks et al. 2008), como apresentado nesta revisão e nas proposições de Tang \& Cheke (2008) e Lima et al. (2009). A introdução da dimensão de manejo integrado na formulação teórica aparece pela adição de termos que representam a intervenção através de inseticidas e/ou pela inclusão de políticas de manejo expressas por monitoramento de níveis populacionais críticos, intervenções periódicas e inclusão da dimensão estocástica no sistema. Os dois últimos itens mencionados aparecem como proposições no presente estudo.

Não há como estabelecer metas para a abordagem teórica de sistemas envolvendo controle de pragas sem levar em conta alguns aspectos importantes para os cenários atuais e futuros em escala global de agroecossistemas. Dentre eles destacamos o confronto entre a demanda crescente de suprimento alimentar e os surtos de pragas ocasionados por mudanças climáticas, os processos de invasão biológica decorrentes da alta mobilidade entre diferentes áreas do planeta nos tempos atuais, a variabilidade genética decorrente do perfil das populações de pragas sujeitas à altas taxas de migração, o histórico de políticas de controle de pragas convencionalmente adotado e a necessidade de implementação de metodologia alternativa para garantir segurança e eficácia em programas de manejo. Além desses aspectos, não se pode deixar de lado as influências causadas por mecanismos biológicos praticamente ainda desconhecidos, advindos da modernização e introdução de ações direcionadas à biotecnologia. Esse cenário diferenciado gera desafios de alta magnitude, indicando a necessidade da implementação de programas de ações multidisciplinares. Neste sentido, a modelagem matemática com ênfase na teoria predadorpresa passa a constituir ferramenta indispensável para a elaboração de programas de manejo de pragas de cunho multidisciplinar.

AGRADECIMENTOS: Os autores desta revisão agradecem o suporte financeiro concedido através de bolsas de estudo e de pesquisa concedidas pela FAPESP e pelo CNPq.

\section{REFERÊNCIAS}

BANKS, H.T.; BANKS, J.E.; JOYNER, S.L. \& STARK, J.D. 2008. Dynamic models for insect mortality due to exposure to insecticides. Mathematical and Computer Modelling, 48: 316332, http://dx.doi.org/10.1016/j.mcm.2007.10.005

COSTA, M.I.S. \& GODOY, W.A.C. 2010. Fundamentos da ecologia teórica. Manole, São Paulo, SP. 66p.

CUSHING, J.M.; COSTANTINO, R.F.; DENNIS, B.; DESHARNAIS, R.A. \& HENSON, S.M. 2003.Chaos in ecology. Academic Press, Amsterdam, 225p.

DE CLERQ, P.; MOHAGHEG, J. \& TIRRY, L. 2000. Effect of host plant on the functional response of the predator Podisus nigrispinus (Heteroptera: Pentatomidae). Biological Control, 18:65-70, http://dx.doi.org/10.1006/bcon.1999.0808

EVANS, E.W. \& TOLLER, T.R. 2007. Aggregation of polyphagous predators in response to multiple prey: ladybirds (Coleoptera: Coccinellidae) foraging on alfalfa. Population Ecology, 49: 29-36, http://dx.doi.org/10.1007/s10144-006-0022-4

HASSEL, M.P. 1984. Inseticides in host-parasitoid interactions. Theoretical Population Biology, 26: 378-386, http://dx.doi. org/10.1016/0040-5809(84)90041-8

HOLLING, C.S. 1965. The functional response of predators to prey density and its role in mimicry and population regulation. Memoirs of the Entomological Society of Canada, 45: 1-60, http://dx.doi.org/10.4039/entm9745fv

KIDD, D. \& AMARASEKARE, P. 2011. The role of transient dynamics in biological pest control: insights from a hostparasitoid community. Journal of Animal Ecology, http://dx.doi. org/10.1111/j.1365-2656.2011.01881.x

KOT, M. 2001. Elements of mathematical ecology. Cambridge University Press, Cambridge, CAM. 453p.

LIMA, E.A.B.F.; FERREIRA, C.P. \& GODOY, W.A.C. 2009.

Ecological Modeling and Pest Population Management: a Possible and Necessary Connection in a Changing World. 
Neotropical Entomology, 38: 699-707, http://dx.doi.org/10.1590/ S1519-566X2009000600001

LOTKA. 1925. Elements of physical biology. Baltimore, Williams and Wilkins Company, 460p., http://dx.doi.org/10.1086/283092

MAY, R.M. \& OSTER, G.F. 1976. Bifurcations and dynamic complexity in simple ecological models. American Naturalist, 110: 573-599.

MURDOCH, W.W.; CHESSON, J. \& CHESSON, P.L. 1985. Biological control in theory and practice. American Naturalist, 125: 344-366, http://dx.doi.org/10.1086/284347

NICHOLSON, A.J. \& BAILEY, V.A. 1935. The balance of animal populations. Proceedings of the Zoological Society of London, 1935: 551-598.

PEDIGO, L.P. \& ZEISS, M.R. 1996. Analyses in insect ecology and management. Iowa State University Press, Ames, IA. 168p.

PROUT, T. \& McCHESNEY, F. 1985. Competition among immatures affects their adult fertility: population dynamics. American Naturalist, 126: 521-558, http://dx.doi. $\operatorname{org} / 10.1086 / 284436$

SCHAALJE, G.B.; STINNER, R.L. \& JOHNSON, D.L. 1989. Modelling insect populations affected by pesticides with application to pesticide efficacy trials. Ecological Modelling, 47: 233-263, http://dx.doi.org/10.1016/0304-3800(89)90003-3

SERRA, H.; SILVA, I.C.R.; MANCERA, P.F.A.; FARIA, L.D.B.; ZUBEN, C.J.V.; ZUBEN, F.J.V.; REIS, S.F. \& GODOY, W.A.C. 2007. Stochastic dynamics in exotic and native blowflies: an analysis combining laboratory experiments and a 2-patch metapopulation model. Ecological research, 22: 686-695, http:// dx.doi.org/10.1007/s11284-006-0322-3

SOUTHWOOD, T.R.E. \& HENDERSON, P.A. 2006. Ecological Methods. Blackwell Science. Victoria, Australia.575p.

TANG, S. \& CHEKE, R.A. 2008. Models for integrated pest control and their biological implications. Mathematical Bioscience, 215: 115-125, http://dx.doi.org/10.1016/j.mbs.2008.06.008

VAN LENTERN, J. C. 2000. Measures of success in biological control of arthropods by augmentation of natural enemies, pp. 77103. In: S. Wratten and G. Gurr (eds). Measures of success in biological control. Kluwer Academic Publishers, Dordrecht, The Netherlands, http://dx.doi.org/10.1007/978-94-011-4014-0_3

VOLTERRA, V. 1926. Fluctuations in the abundance of a species considered mathematically. Nature, 118: 558-560, http://dx.doi. org/10.1038/118558a0
ZHANG, H.; GEORGESCU, P. \& CHEN, L. 2008. On the impulsive controllability and bifurcation of a predator-pest model of IPM. Biosystems, 93: 151-171, http://dx.doi.org/10.1016/j. biosystems.2008.03.008

Submetido em 01/08/2011

Aceito em 03/10/2011 